\title{
Fast Multipole Acceleration of a MoM Code for the Solution of Composed Metallic/Dielectric Scattering Problems
}

\author{
U. Jakobus and J. van Tonder \\ EM Software \& Systems - S.A. (Pty) Ltd, PO Box 1354, Stellenbosch 7599, South Africa
}

\begin{abstract}
An existing method of moments (MoM) code for the solution of complex scattering bodies has been accelerated by means of a multilevel fast multipole method (MLFMM). We demonstrate the usage of this technique both for metallic structures (wires and surfaces) and for dielectric bodies (volume and surface equivalence principle). Aspects like the effect of the type of integral equation, preconditioning schemes, or iterative solution techniques are discussed. But also limitations are addressed, which are encountered when for instance attempting to model highly lossy dielectric bodies with a high permittivity. Several validation and application examples demonstrate the usefulness of this method, both with regard to the obtained accuracy, but also with respect to the potential saving in memory and run-time as compared to a standard MoM formulation.
\end{abstract}

\section{Introduction to the Multilevel Fast Multiple Method (MLFMM)}

\subsection{Formulation of the MLFMM}

A brief outline of the MLFMM will be presented in this section. The interested reader is referred to Coifman et al. (1993); Song and Chew (1994, 1995); Chew et al. (1997); Song et al. (1997); Gyure and Stalzer (1998); Chew et al. (2001) for more details.

The MLFMM is is based on a hierarchical grid. At the top level (level 0) the whole computational space is enclosed by one large cube. At the next level (level 1) this cube is then subdivided in 3-dimensions into a maximum of 8 child cubes. This process is repeated until at the finest level the cube side length is approximately $0.25 \lambda$. Only non-empty cubes are stored at each level forming a tree-like data structure. Figure 1 shows the cubes at the finest level for one automotive example.

Correspondence to: U. Jakobus

(jakobus@emss.co.za)
In the MoM framework a system of linear equations

$Z I=V$

needs to be solved. The MLFMM is implemented by writing the impedance matrix $Z$ into the near-field term $Z_{\text {near }}$ and the far-field term $Z_{f a r}$, i.e. Equation (1) then becomes

$Z_{\text {near }} I+Z_{\text {far }} I=V$.

$Z_{\text {near }}$ consists of all matrix elements where basis and weighting functions are within the same box or in adjacent boxes at the finest level. Only this near-field mattrix $Z_{\text {near }}$ is computed traditionally and stored in a sparse format. The system of linear equations (1) is solved with an iterative technique where matrix-vector products $Z I_{k}$ are required, with $k$ indicating the iteration counter. The far-field term $Z_{f a r}$ is never computed explicitly, but the matrix-vector product $Z_{f a r} I_{k}$ is computed via the MLFMM as

$Z_{\text {far }} I_{k}=D T A I_{k}$

with the following three phases:

- The Aggregation phase where all the basis functions inside the same source cube (at the finest level) are grouped together,

- The Translation phase from the source cube to the observation cube,

- The Disaggregation phase from the centre of the observation cube to every basis function inside that cube.

All these phases make use of the addition theorem to approximate the free-space Green's function

$$
\begin{aligned}
& G\left(\overline{\mathbf{x}}, \overline{\mathbf{x}}^{\prime}\right)=\frac{e^{-j k R}}{R} \approx \\
& \frac{-j k}{4 \pi} \int d^{2} \hat{\mathbf{k}} e^{-j \overline{\mathbf{k}} \cdot\left(\overline{\mathbf{x}}-\overline{\mathbf{x}}_{m}\right)} T_{L}\left(\overline{\mathbf{k}}, \overline{\mathbf{X}}_{m^{\prime} m}\right) e^{+j \overline{\mathbf{k}} \cdot\left(\overline{\mathbf{x}}^{\prime}-\overline{\mathbf{x}}_{m^{\prime}}\right)}
\end{aligned}
$$




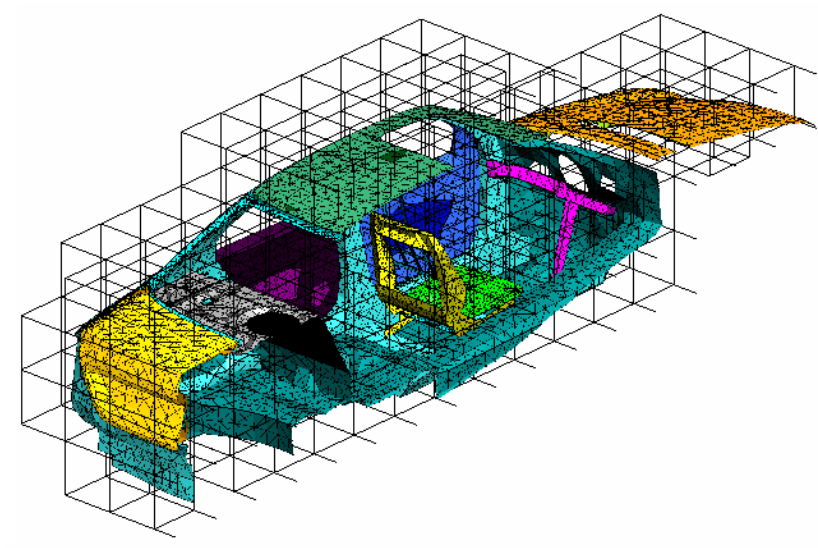

Fig. 1. The MLFMM boxes at the finest level for one automotive example. Only half of the geometry is shown for clarity.

where

$T_{L}\left(\overline{\mathbf{k}}, \overline{\mathbf{X}}_{m^{\prime} m}\right)=\sum_{l=0}^{L}(-j)^{l}(2 l+1) h_{l}^{(2)}\left(k X_{m^{\prime} m}\right) P_{l}\left(\hat{\mathbf{k}} \cdot \hat{\mathbf{X}}_{m^{\prime} m}\right)$

and the number of terms $L$ are determined empirically for a given accuracy $\varepsilon$ by the formula

$L=k D+1.8(k D)^{1 / 3}\left(\log _{10}(1 / \varepsilon)\right)^{2 / 3}$

with the wavenumber $k$ and the box size $D$.

For the integration over the sphere a quadrature rule with $2 L^{2}$ points is applied according to Coifman et al. (1993); Song and Chew (1994). Looking at Eq. (4) the aggregation step is given by $e^{+j \overline{\mathbf{k}} \cdot\left(\overline{\mathbf{x}}^{\prime}-\overline{\mathbf{x}}_{m^{\prime}}\right)}$, the translation step by $T_{L}\left(\overline{\mathbf{k}}, \overline{\mathbf{X}}_{m^{\prime} m}\right)$, and the disaggregation step by $e^{-j \overline{\mathbf{k}} \cdot\left(\overline{\mathbf{x}}-\overline{\mathbf{x}}_{m}\right)}$.

Eqation (2) must be solved with iterative techniques (for example CGS, Bi-CGSTAB, etc.) since we only have the sparse $Z_{\text {near }}$ and never store $Z_{f a r}$. For general open structures the Electric Field Integral Equation (EFIE) is poorly conditioned, causing the iterative technique to converge very slowly (or even diverge). To accelerate the rate of convergence we use a preconditioner that is computed from the near-field matrix $Z_{\text {near }}$. Implemented preconditioners include Incomplete LU (ILU), Block-Jacobi and Block-Jacobi one-level-up.

\subsection{Scaling of memory and CPU-time}

Let $N$ be the number of unknowns (i.e. number of basis functions). The traditional MoM scales as $N^{2}$ in terms of memory (to store the impedance matrix) and as $N^{3}$ in terms of CPU-time (to solve the linear set of equations). When $N$ becomes large the MoM will therefore require too much memory and CPU-time. Much more favourable is the MLFMM, which scales as $N \log N$ in memory and as $N \log ^{2} N$ in terms of CPU-time. Figure 2 shows typical memory and CPUtimes for an automotive example (full vehicle of approximate length $4.5 \mathrm{~m}$ including seats, windows, etc. at different frequencies). One can see that the actual values for the
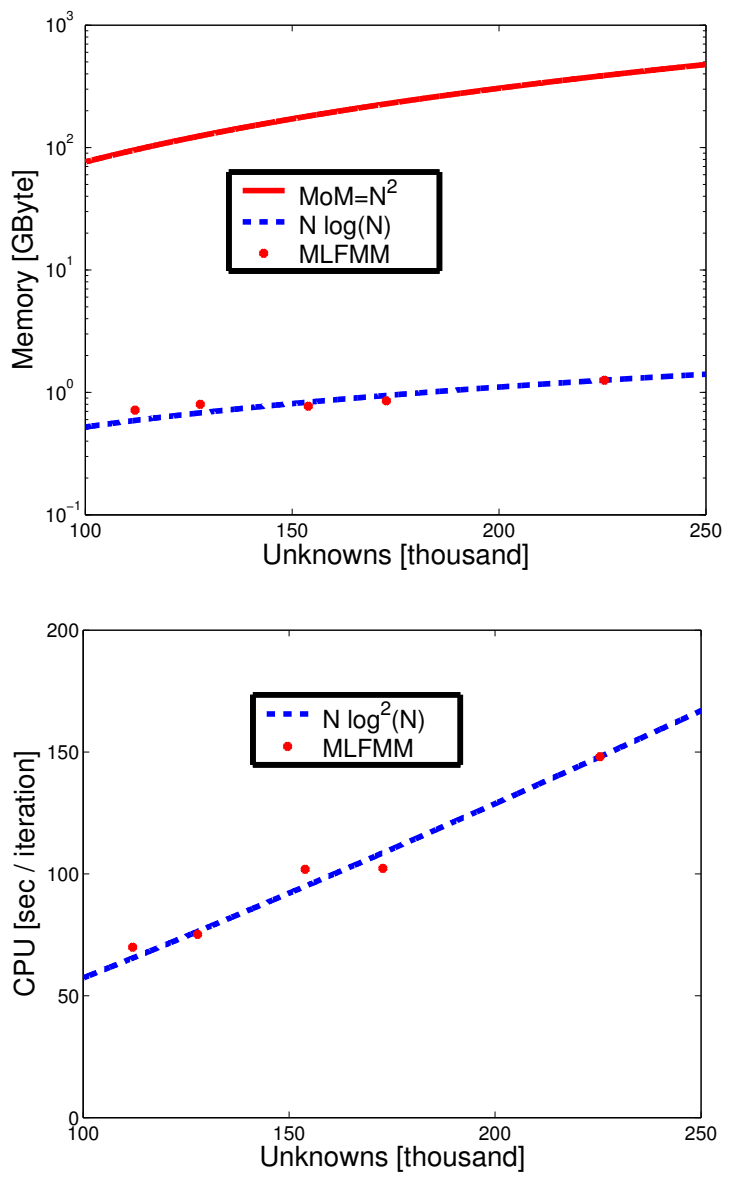

Fig. 2. Memory and CPU-time scaling for the MLFMM for an automotive example.

Table 1. Typical memory requirement and CPU-times for an antenna analysis on an aircraft. All MLFMM runs performed on a 64-bit AMD Opteron 248 (2.2 GHz).

\begin{tabular}{cccc}
\hline $\begin{array}{c}\text { No. } \\
\text { of unknowns }\end{array}$ & $\begin{array}{c}\text { MoM [GByte] } \\
\text { [GByte] }\end{array}$ & $\begin{array}{c}\text { MLFMM } \\
\text { [GByte] }\end{array}$ & $\begin{array}{c}\text { MLFMM } \\
\text { [hours] }\end{array}$ \\
\hline 1030891 & 16216 & 10.3 & 15.8 \\
1573620 & 37785 & 13.9 & 21.8 \\
\hline
\end{tabular}

MLFMM (dots) follow nicely the theoretically expected scaling (dashed lines).

The advantages of the MLFMM become more evident as the geometry becomes larger in terms of the wavelength. The results for an aircraft are given in Table 1. The MLFMM for the 1.57 million unknowns case uses 2718 times less memory than the MoM.

\subsection{Implementation details}

Our MLFMM implementation in the computer code FEKO (see FEKO, 2004) includes amongst others the following 

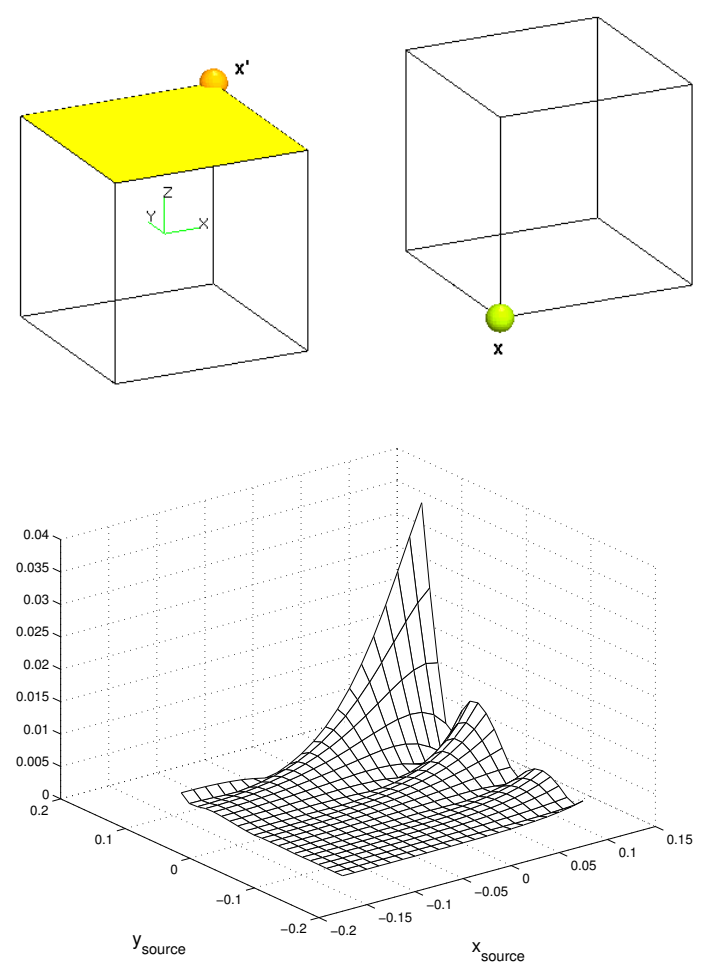

Fig. 3. Relative error in the MLFMM representation of the freespace Green's function as a function of source/observer distance for a fixed number of terms $L$.

features, all of which were extensively verified:

- EFIE (valid for general open geometries) for metallic triangular surface patches with basis functions according to Rao et al. (1982),

- Metallic wires,

- Connection basis functions between wires and triangles,

- The combined field integral equation (CFIE) (only valid for closed geometries),

- Dielectric cuboid elements with the volume equivalence principle,

- Dielectric bodies with the surface equivalence principle using the PMCHW formulation,

- The geometry can be located above real ground,

- Thin dielectric sheet approximation to model e.g. thin windows.

It should be mentioned that both the CFIE and the volume equivalence principle result in Fredholm integral equations of the second kind with excellent convergence during the iterative solution. Therefore when we use the CFIE or volume cuboids, then we can use a smaller preconditioner

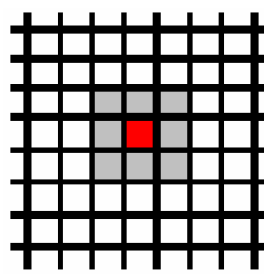

1 buffer box

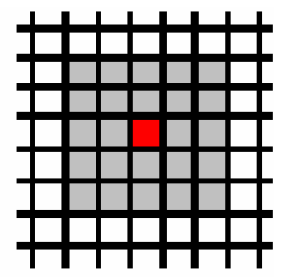

2 buffer boxes

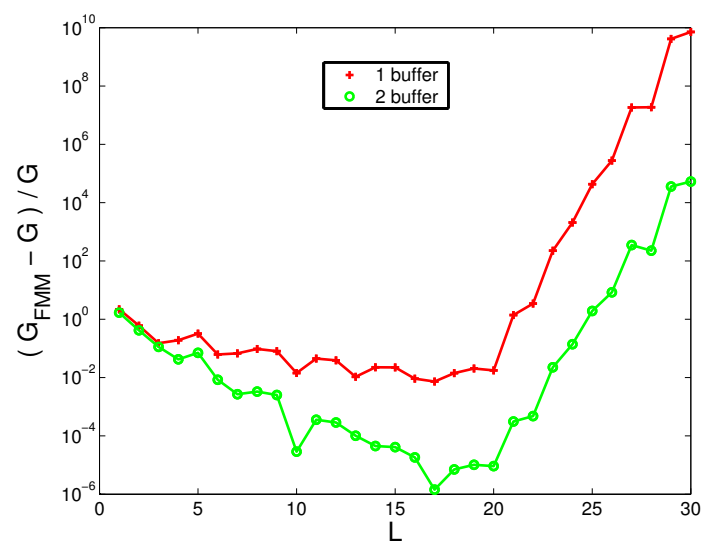

Fig. 4. Using 1 or 2 buffer boxes in the MLFMM gridding and associated error in the Green's function versus the number of terms $L$.

(or even no preconditioner). To obtain a small preconditioner one can use the Block-Jacobi preconditioner (blockdiagonal obtained from boxes at finest level), or the BlockJacobi one-level-up (obtained from the parent boxes of the Block-Jacobi). Reducing the level-of-fill of the ILU preconditioner also reduces the size of the preconditioner. For general open geometries the EFIE is poorly conditioned and requires a good preconditioner (typically an ILU with the level-of-fill=12 is used). The Bi-CGSTAB iterative solver outperformed the other solvers (CGS, RGMRES, etc.) in most of our applications.

\section{Considerations regarding the treatment of dielectric bodies}

For a fixed value of $L$ in Eq. (5), the error between the exact Green's function and the MLFMM approximation in Eq. (4) is computed in a plane in Fig. 3. It can be seen that the maximum error corresponds to the situation when the source and observation points are located at the corners as indicated by the spheres ((see also Ohnuki and Chew, 2003).

The empirical formula to determine $L$ in Eq. (6) is no longer valid for large dielectric losses. $L$ must then be determined numerically (Geng et al., 2001, Fig. 2) at each level in the MLFMM tree so that the maximum error is below the required threshold. However, if $L$ becomes too large the Hankel function in Eq. (5) will diverge for large order and small argument. 

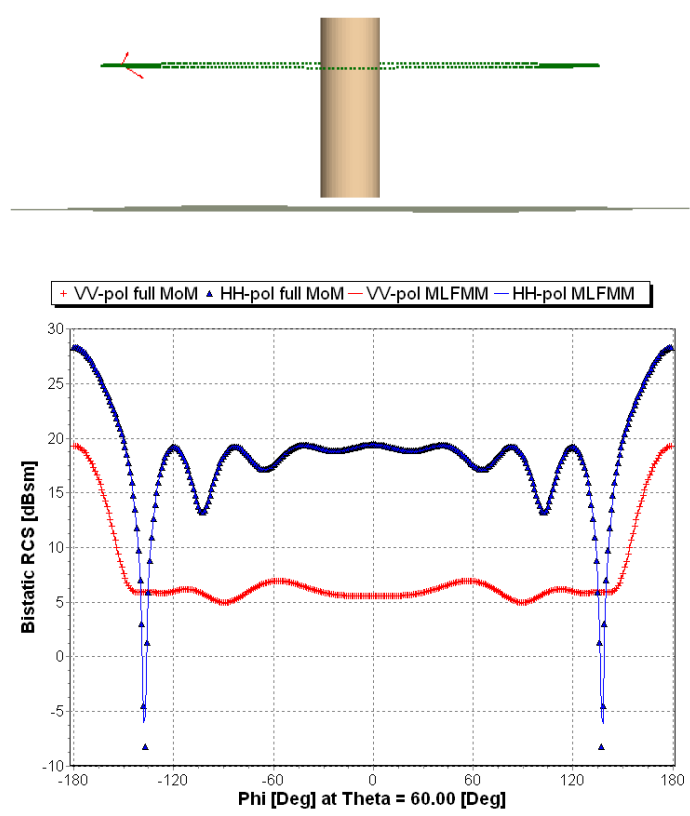

Fig. 5. Bistatic RCS computation of a metallic cylinder above real ground.

One trick which can be used here is to increase the nearfield matrix by so-called buffer boxes, so that for the far-field terms the minimum distances where the representation (4) is used are larger.

The lower bound on the argument of the Hankel function is dependent on the number of buffer boxes. By increasing the number of buffer boxes from one to two, it can be seen in Fig. 4 that the error decreases for a fixed $L$. Therefore, if for a fixed buffer box size the numerically computed error remains above the required threshold, then the number of boxes must be increased. The drawback is that the size of the near field matrix increases dramatically with the number of buffer boxes.

As example, consider human eye tissue with typical permittivity $\varepsilon_{r}=55-j 23$. The required maximum error shall be smaller than $10^{-3}$, and for a box size of $0.5 \lambda_{0}$ in the MLFMM tree we determine numerically that we need two buffer boxes and $L=70$. For comparison, with the same box size and buffer boxes, the free-space case $\varepsilon_{r}=1$ will need only $L=12$. Since the integration over the unit Ewald sphere in Eq. (4) uses a quadrature rule with $2 L^{2}$ points, the eye will use 34 more sample points. Therefore, as the dielectric loss increases the MLFMM will become slower and use more memory.

\section{Application and validation examples}

The MLFMM has been validated extensively with analytical, published, as well as full MoM results. In this section some verification examples shall be presented and discussed.
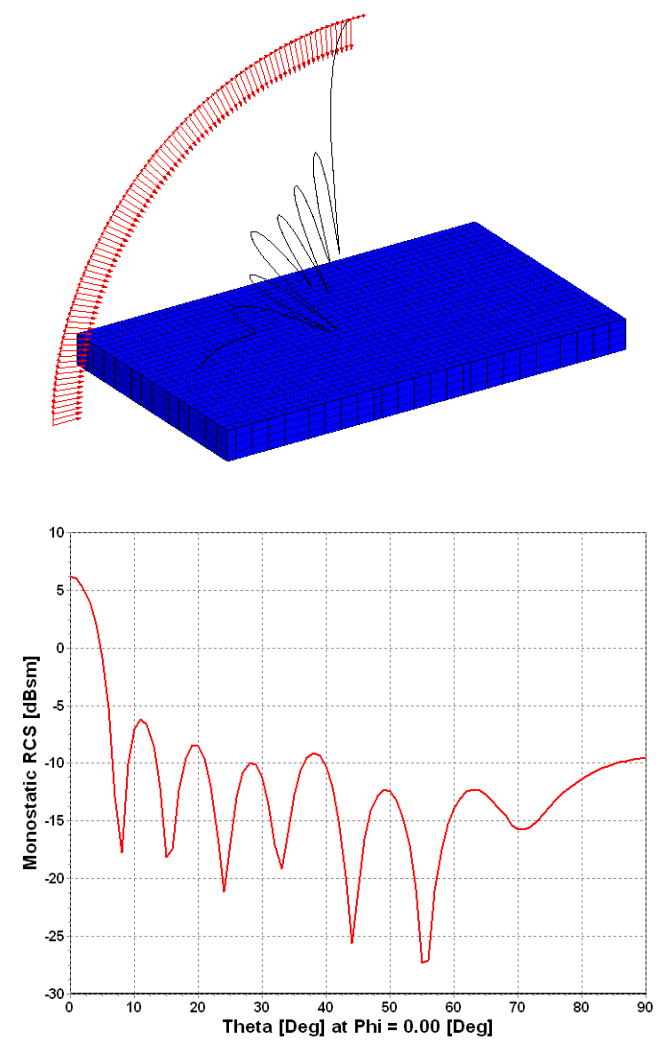

Fig. 6. Monostatic RCS from a dielectric slab computed with the MLFMM using the volume equivalence principle.

To verify the implementation of objects above real ground consider the cylinder located above earth in Fig. 5. The cylinder is of height $3 \mathrm{~m}$ and diameter $1 \mathrm{~m}$ situated $0.2 \mathrm{~m}$ above ground with complex permittivity $\varepsilon_{r}=6.5-j 0.6$. A plane wave is incident from $\vartheta_{i n c}=30^{\circ}$ and $\varphi_{i n c}=0^{\circ}$. The bistatic RCS shall be computed versus the angle $\varphi_{\text {scat }}$ for $\vartheta_{\text {scat }}=60^{\circ}$ and at a frequency of $f=600 \mathrm{MHz}$.

This example is relatively small and the cylinder consists of 6168 metallic triangles resulting in 9252 unknowns. In Fig. 5 the bistatic RCS is depicted for the full MoM (1306 MByte memory) and also for the MLFMM (231 MByte memory). Excellent agreement can be observed between the MoM, MLFMM and the published results in Geng et al. (2000) and $\mathrm{Hu}$ and Chew (2001) (not shown in the graph).

Dielectric cuboids treated with the volume equivalence principle have been implemented in the MLFMM and verified to published results. In Fig. 6 the monostatic RCS of a dielectric slab computed with the MLFMM agrees very well with that published in Chew et al. (2001, Fig. 11.16, pp. 522). The dimensions of the slab are $3.5 \lambda_{0} \times 2 \lambda_{0} \times 0.25 \lambda_{0}$. The frequency is $1 \mathrm{GHz}$ and the permittivity is $\varepsilon_{r}=3-j 0.09$.

To validate the MLFMM for more complex real-life problems we will compare results to those obtained using the full MoM for a mobile phone radiating inside a Lancia car model. The model in Fig. 7 is divided into 20754 triangles 


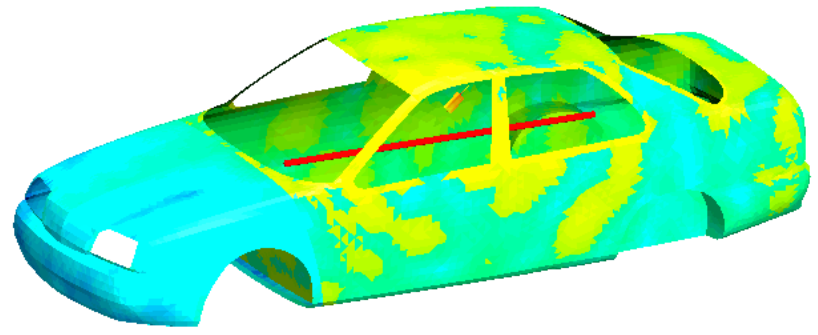

Fig. 7. Analysis of a mobile phone radiating inside a Lancia car model at $600 \mathrm{MHz}$.
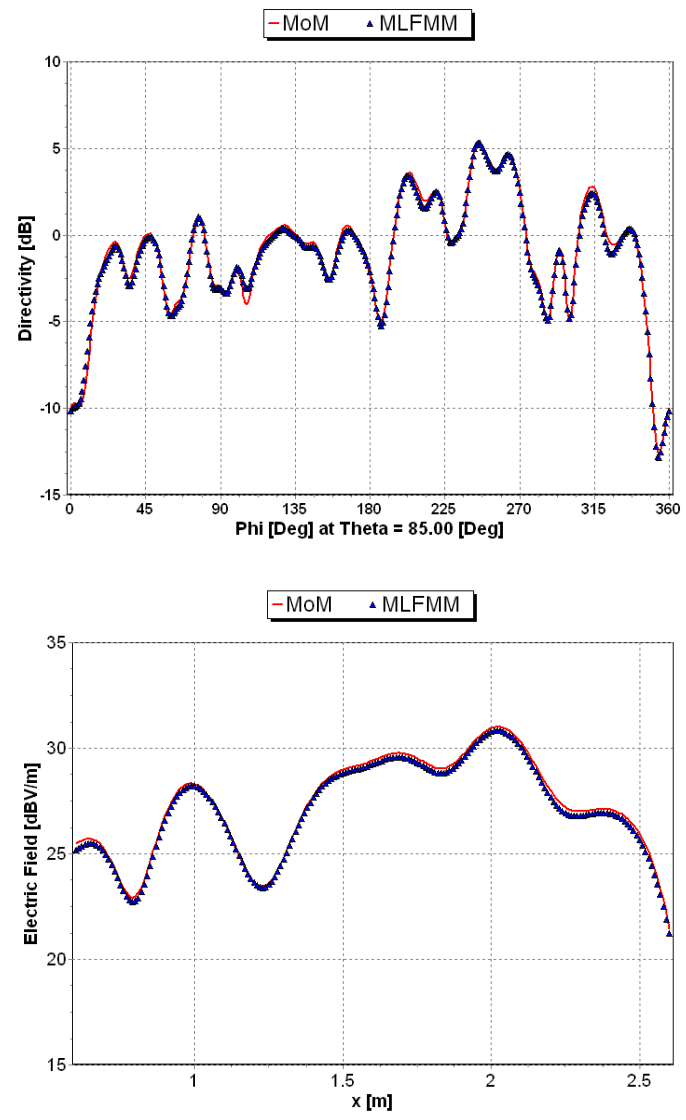

Fig. 8. The far field (top) and near field (bottom) of a mobile phone inside the Lancia car model as shown in Fig. 7.

and 9 wire segments resulting in 30915 unknown basis functions. This is a relatively small example, but the full MoM already requires 14583 MByte of memory. The MoM run was done on a Linux cluster of 16 processors (AMD Athlon $1 \mathrm{GHz}$ ) and the sum of the CPU-times of the 16 processes is $33.17 \mathrm{~h}$ ( $2.073 \mathrm{~h}$ on average per process). The MLFMM requires only 443 MByte of memory and $8.4 \mathrm{~min}$ of CPU-time on a single Intel P4 $2.4 \mathrm{GHz}$ processor.

Very good agreement between the full MoM and the MLFMM can be seen in Fig. 8, for both the far field and the near field. The far field is computed versus $\varphi$ at $\vartheta=85^{\circ}\left(5^{\circ}\right.$
Table 2. Memory requirements and CPU-times for a dielectrically coated sphere.

\begin{tabular}{ccc}
\hline Method & Memory [GByte] & Runtime [hours] \\
\hline MoM & 9.43 & 3.269 \\
MLFMM & 1.00 & 0.734 \\
FEM & 3.04 & 0.388 \\
\hline
\end{tabular}
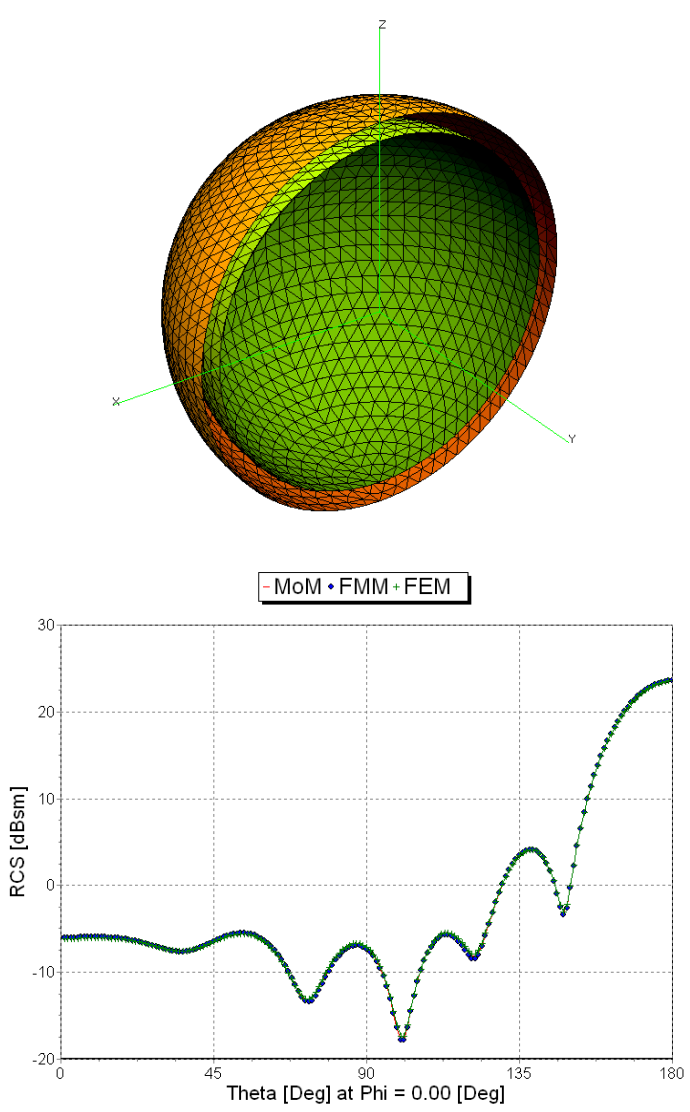

Fig. 9. Bistatic RCS of a dielectrically coated sphere.

above the horizon). The near field is computed along a line inside the Lancia as shown in Fig. 7.

As an example for lossy dielectric structures, we consider the dielectrically coated sphere shown in Fig. 9. This example was also presented by Sertel and Volakis (2004). The inner sphere has a diameter $d_{i n}=1.8 \lambda_{0}$ and permittivity $\varepsilon_{r}^{i n}=1.75-j 0.3$. The outer sphere has $d_{\text {out }}=2.0 \lambda_{0}$ and $\varepsilon_{r}^{\text {out }}=1.25-j 1.25$ (thus loss tangent of one). The surface equivalence principle is used in the MoM and MLFMM with the number of unknowns $N=25152$. Memory and run-times (no symmetry used in all cases) are given in Table 2. Figure 9 shows the results for the MoM, MLFMM and also a reference solution using FEM (Finite Element Method). It can be seen that the agreement between the three techniques is excellent. 


\section{Conclusions}

We have shown that with the MLFMM large complex electromagnetic problems can be solved using only a fraction of the memory and CPU-time as required by the full MoM. The errors introduced by the MLFMM are fully controllable unlike with other asymptotic techniques as Physical Optics (PO) or Uniform Theory of Diffraction (UTD). This enables the MLFMM to produce very accurate results. Furthermore, the formulation and our implementation have the advantage that the whole gridding and split into near and far field matrices are done automatically. This eliminates any a priori decision by the user to decide what should be in the near field or the far field.

We have demonstrated the usage of this technique both for metallic structures (wires and surfaces) and for dielectric bodies (volume and surface equivalence principle). We have also highlighted the difficulties encountered when attempting to model highly lossy dielectric bodies, and we have presented some solution strategies involving buffer boxes.

\section{References}

Chew, W. C., Jin, J. M., Lu, C. C., Michielssen, E., and Song, J. M.: Fast solution methods in electromagnetics, IEEE Transactions on Antennas and Propagation, 45, 533-543, 1997.

Chew, W. C., Jin, J. M., Michielssen, E., and Song, J. M.: Fast and efficient algorithms in computational electromagnetics, Artech House, Boston, 2001.

Coifman, R., Rohklin, V., and Wandzura, S.: The fast multipole method for the wave equation: A pedestrian prescription, IEEE Transactions on Antennas and Propagation, 35, 7-12, 1993.

FEKO: Field Computations Involving Bodies of Arbitrary Shape, EM Software \& Systems - S.A. (Pty) Ltd, Stellenbosch, South Africa, http://www.feko.info, 2004.
Geng, N., Sullivan, A., and Carin, L.: Multilevel fast-multipole algorithm for scattering from conducting targets above or embedded in a lossy half space, IEEE Transactions on Geoscience and Remote Sensing, 38, 1561-1573, 2000.

Geng, N., Sullivan, A., and Carin, L.: Fast multipole method for scattering from an arbitrary PEC target above or buried in a lossy half space, IEEE Transactions on Antennas and Propagation, 49, 740-748, 2001.

Gyure, M. F. and Stalzer, M. A.: A prescription for the multilevel Helmholtz FMM, IEEE Computational Science \& Engineering, 5, 39-47, 1998.

Hu, B. and Chew, W. C.: Fast inhomogeneous plane wave algorithm for scattering from objects above the multilayered medium, IEEE Transactions on Geoscience and Remote Sensing, 39, 10281038, 2001.

Ohnuki, S. and Chew, W. C.: Numerical accuracy of multipole expansion for 2-D MLFMA, IEEE Transactions on Antennas and Propagation, 51, 1883-1890, 2003.

Rao, S., Wilton, D., and Glisson, A.: Electromagnetic scattering by surface of arbitrary shape, IEEE Transactions on Antennas and Propagation, 30, 409-418, 1982.

Sertel, K. and Volakis, J.: Multilevel fast multipole method solution of volume integral equations using parametric geometry modeling, IEEE Transactions on Antennas and Propagation, 52, 16861692, 2004.

Song, J. M. and Chew, W. C.: Fast multipole method solution using parametric geometry, Microwave and Optical Technology Letters, 7, 760-765, 1994.

Song, J. M. and Chew, W. C.: Multilevel fast-multipole algorithm for solving combined field integral equations of electromagnetic scattering, Microwave and Optical Technology Letters, 10, 1419, 1995.

Song, J. M., Lu, C. C., and Chew, W. C.: Multilevel fast multipole algorithm for electromagnetic scattering by large complex objects, IEEE Transactions on Antennas and Propagation, 45, 1488-1493, 1997. 